\title{
Exchange mechanism for electron paramagnetic resonance of individual adatoms
}

\author{
J. L. Lado, ${ }^{1}$ A. Ferrón, ${ }^{2}$ and J. Fernández-Rossier ${ }^{1,3}$ \\ ${ }^{1}$ QuantaLab, International Iberian Nanotechnology Laboratory (INL), Avenida Mestre Jos Veiga, 4715-330 Braga, Portugal \\ ${ }^{2}$ Instituto de Modelado e Innovación Tecnológica (CONICET-UNNE) and Facultad de Ciencias Exactas, Naturales y Agrimensura, \\ Universidad Nacional del Nordeste, Avenida Libertad 5400, W3404AAS Corrientes, Argentina \\ ${ }^{3}$ Departamento de Física Aplicada, Universidad de Alicante, 03690 Spain
}

(Received 10 November 2016; revised manuscript received 31 August 2017; published 13 November 2017)

\begin{abstract}
We propose a universal mechanism that makes it possible to drive an individual atomic spin using a spinpolarized scanning tunnel microscope (STM) with an oscillating electric signal. We show that the combination of the distance-dependent exchange with the magnetic tip and the electrically driven mechanical oscillation of the surface spins permits us to control their quantum state. Based on a combination of density functional theory and multiplet calculations, we show that the proposed mechanism is essential to account for the recently observed electrically driven paramagnetic spin resonance (ESR) of an individual Fe atom on a $\mathrm{MgO} / \mathrm{Ag}(100)$ surface. Our findings set the foundation to deploy the ESR-STM quantum sensing technique to a much broader class of systems.
\end{abstract}

DOI: 10.1103/PhysRevB.96.205420

\section{INTRODUCTION}

Scanning tunneling microscopy (STM) and electron paramagnetic resonance (EPR) are two very powerful experimental techniques whose integration has been pursued in the past three decades [1-6] and motivated a substantial body of theoretical work [7-9]. In EPR, spin transitions are excited with an ac field that permits us to resolve spin excitation with a resolution limited by the intrinsic spin relaxation broadening of the species. In continuous wave (cw) EPR, this can be down to a few MHz for amorphous hydrogenated silicon [10]. However, standard detection techniques based on induction require probing at least $10^{7}$ spins [11] and have therefore a very poor spatial resolution. In contrast, STM permits us to probe individual atoms with an exquisite spatial resolution, but when it comes to performing spin spectroscopy, it relies on inelastic electron tunneling [12] (IETS), whose spectral resolution is limited [13] by $5.4 k_{B} T$, where $T$ is the temperature. Thus, even for the coldest STM so far [14], the spectral resolution of IETS spectroscopy would be above $30 \mathrm{GHz}$, i.e., three orders of magnitude worse off than cw-EPR.

In a recent experimental breakthrough, Baumann et al. [6] have reported the measurement of the electron paramagnetic resonance of an individual $\mathrm{Fe}$ atom deposited on top of an atomically thin $\mathrm{MgO}$ layer grown on $\mathrm{Ag}(001)$, using a spinpolarized STM tip (see Fig. 1) to both drive the atom with an ac signal and to probe the resulting reaction. For the driving, they applied a radio frequency (rf) voltage $V_{R F}$ across the tip sample with frequency $f$. The resulting change in dc current, $I_{D C}$, as a function of $f$ displayed a very narrow (3 MHz) resonance peak, at the frequency $f_{0}$ that matches the Zeeman splitting of the magnetic adatom ground state doublet $(26 \mathrm{GHz}$ for $B_{z}=0.2 \mathrm{~T}$ ). The peak, well above the noise level, would shift upon application of a magnetic field, making it possible thereby to detect $50 \mu T$ variations with subatomic resolution.

The experiment of Baumann et al. [6], electrically driven paramagnetic spin resonance (ESR), outperforms the spectral resolution of IETS-STM spectroscopy by four orders of magnitude, at the same temperature, and reaches the absolute detection limit by probing a single spin. The recently reported application of this remarkable setup to probe the magnetic moment of individual atoms nearby $[15,16]$ demonstrates the potential of ESR-STM technique as an extremely versatile quantum sensing tool.

In this paper, we address a fundamental question that begs for an answer in order to understand the working principles of any STM-ESR setup, namely, how an rf voltage can drive the atomic spin. Baumann et al. [6] proposed a mechanism that combines two ingredients. First, the rf electric field induces a mechanical oscillation $z(t)$ of the surface atom. Second, the induced modulation of the crystal field, combined with the spin-orbit interaction of the $d$ electrons of the surface atom, results in transitions between the two lowest energy levels of the atomic spin. Whereas the first ingredient applies for any charged surface atom, the second is only valid for the specific symmetry of the $\mathrm{Fe} / \mathrm{MgO}$ system.

Here we propose an alternative universal mechanism that permits us to drive the spin of a charged surface atom, using an rf electrical voltage and an STM tip with a magnetic atom in the apex. The mechanism is based on the notion that spin interactions between the tip and the surface atom depend strongly on their distance. The electric modulation of the surface atom position results in a variation of the spin-spin interaction that can efficiently drive the surface spin. This changes the occupation of the surface spin states, and that changes its average magnetic moment. Because of the spin-polarized nature of the STM tip, this leads to a magnetoresitve change of the $d c$ current.[6] In the present paper, we focus on the nature of the coupling between the ac voltage and the surface spin, without trying to evaluate the $\mathrm{dc}$ current itself.

The paper is organized as follows. In Sec. II, we present the different mechanism capable of yielding a Rabi coupling, In Sec. III, we present our microscopic modeling of the experimental system [6], and the symmetry difference that allows us to distinguish between the exchange and crystal field mechanisms. Finally, in Sec. IV, we summarize our conclusions. 

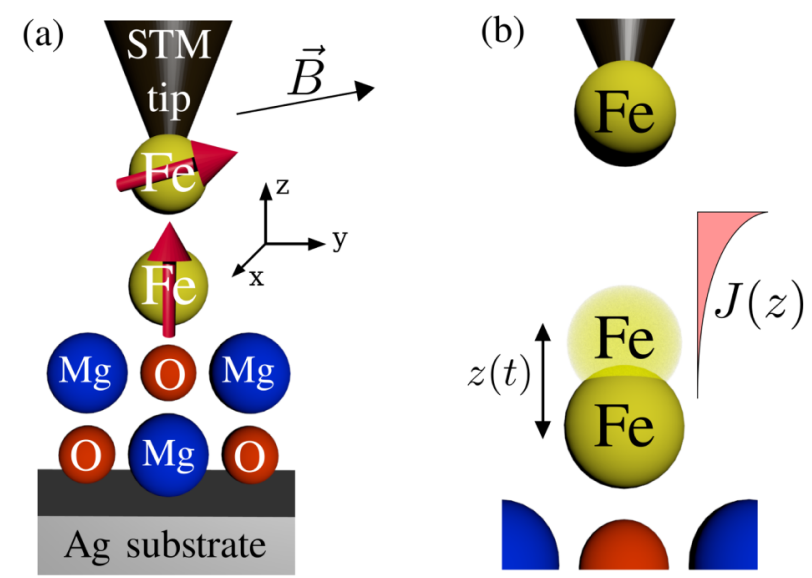

(c)

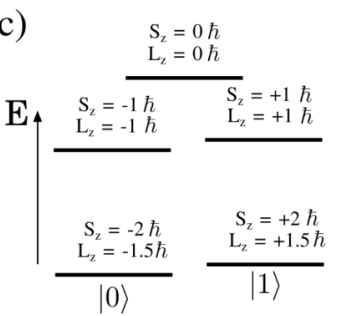

(d)

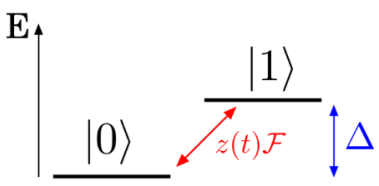

FIG. 1. (a) Sketch of an $\mathrm{Fe}$ atom in $\mathrm{MgO}$, where the ESR signal will be measured with an STM tip. The electric field created by the tip moves the Fe atom upward and downward as shown in panel (b), in particular changing the exchange interaction $J(z)$. Panel (c) shows a sketch of the energy levels of $\mathrm{Fe}$ on $\mathrm{MgO}$ in the presence of a small off-plane magnetic field $\left(B_{z}=0.2 \mathrm{~T}\right)$. Panel $(\mathrm{d})$ shows the reduced low-energy Hamiltonian, restricting the dynamics to the two lowest energy levels in panel (c).

\section{ELECTRICALLY DRIVEN SPIN EXCITATION MECHANISMS}

When a voltage difference $V_{\mathrm{RF}}(t)$ is applied across the gap between the tip and the sample, the electric field induces a small vertical displacement of the surface atom $z(t)$ [see Fig. 1(b)]. We can Taylor expand the spin Hamiltonian of the surface atom around $z=0$, the surface atom equilibrium position:

$$
\mathcal{H} \approx \mathcal{H}_{0}+\left.z(t) \frac{\partial \mathcal{H}}{\partial z}\right|_{z=0}
$$

The previous Hamiltonian consists of a time-independent term $\mathcal{H}_{0}$ and a time-dependent term $\left.z(t) \frac{\partial \mathcal{H}}{\partial z}\right|_{z=0}$. The first term determines the excitation spectra of the quantum system. When the second term is modulated at a frequency that matches the energy splitting $\Delta$ between a given pair of eigenstates $(|M\rangle$ and $|N\rangle$ ) of the atomic spin Hamiltonian $\mathcal{H}_{0}$, transitions will be induced provided that the Rabi force $\mathcal{F}_{\mathcal{N}, \mathcal{M}}=\left\langle N\left|\frac{\partial \mathcal{H}}{\partial z}\right| M\right\rangle \neq$ 0 . Several terms in the Hamiltonian can yield a nonzero contribution to the term $\frac{\partial \mathcal{H}}{\partial z}$, whose physical meaning is related to how the Hamiltonian felt by the surface spin changes under small displacements of the surface atom and is responsible for coupling the surface spin to an electrical signal.

\section{A. Exchange-driven mechanism}

Here we propose that the variation on the tip-surface distance provides such coupling in the form of exchange interaction $\mathcal{H}_{J}=J(z(t)) \vec{S}_{T} \cdot \vec{S}$. Such contribution, already studied theoretically and observed experimentally [17-22], will be present in any surface spin when probed with a spinpolarized STM and therefore represents a universal mechanism for electron paramagnetic resonance of individual adatoms.

We ignore the quantum fluctuations of the magnetic moment of the apex atom, quenched by the combination of an applied magnetic field and strong Korringa damping with the tip electron bath. Therefore, we treat the tip spin in a mean field or classical approximation, following Yan et al. [20], and replace $\vec{S}_{T}$ by its statistical average $\left\langle\vec{S}_{T}\right\rangle$. For the sake of simplicity, we will restrict the discussion to the case when the dynamics is restricted to the two lowest energy states $|0\rangle$ and $|1\rangle$, although our description of the atomic spin states includes hundreds of multielectron configurations, as we describe below [23,24]. Within the two-level approximation, the relevant operator for the Rabi force associated with the exchange interaction is

$$
\mathcal{F}_{J}=\frac{\partial J(z)}{\partial z}\left\langle\vec{S}_{T}\right\rangle \cdot\langle 0|\vec{S}| 1\rangle
$$

This mechanism does not rely on the specifics of the crystal field of the adatom or on spin-orbit coupling, suggesting the possibility of applying the single-spin STM-ESR to a variety of systems, including $S=1 / 2$ atoms and light element magnetism [25].

\section{B. Crystal-field-driven mechanism}

A different mechanism was proposed by Baumann et al. [6] specific for $\mathrm{Fe}$ on the (100) $\mathrm{MgO}$ surface, where the combination of crystal field and spin-orbit interaction would couple the two lowest energy levels of the atomic spin. The relevant Rabi force for this crystal field mechanism reads

$$
\mathcal{F}_{C F}=\frac{\partial F_{W}}{\partial z}\left\langle 0\left|l_{x}^{4}+l_{y}^{4}\right| 1\right\rangle,
$$

where $F_{W}$ is a crystal field parameter and $l_{x}$ and $l_{y}$ are the single-particle orbital momentum operator for the $d$ electrons of the surface Fe.

\section{Dipolar-coupling-driven mechanism}

A third mechanism is provided by the dipolar interaction between the magnetic moment of the tip and the Fe atom on the surface, where the tip creates an $z$-dependent in-plane magnetic field, giving rise a dipolar mixing term of the form

$$
\mathcal{F}_{\text {dip }}=\mu_{B} \frac{\partial B_{x}^{\text {Tip }}}{\partial z}\left\langle 0\left|L_{x}+2 S_{x}\right| 1\right\rangle,
$$

where $B_{x}^{\text {Tip }}=2 \frac{\mu_{0}}{4 \pi} \frac{\mu_{B} S_{x}^{T i p}}{d_{\mathrm{Fe}-\mathrm{Tip}}^{3}}$ is the magnetic field created by the tip, assuming the moment of the tip lies in plane and $d_{\mathrm{Fe}-\mathrm{Tip}}^{3}$ is the tip-Fe distance. 


\section{Mechanical coupling between electrical signal and local spin}

For the three mechanisms, the resulting two-level system in the $|0\rangle,|1\rangle$ subspace can be written as $\mathcal{H}_{\text {eff }}=\left(\begin{array}{cc}\frac{\Delta}{2} & \hbar \Omega(t) \\ \hbar \Omega(t) & -\frac{\Delta}{2}\end{array}\right)$, with $\Delta=\hbar \omega_{0}$ beingthe splitting between the ground state and the first excited state, and the driving Rabi term is

$$
\Omega(t)=\frac{\mathcal{F}}{\hbar} z(t) .
$$

Because of the oscillating electrical signal applied, $z(t)=$ $z_{0} \cos \omega t$, with $z_{0} \propto V_{R F}(t)$, we can write down $\Omega(t)=$ $\Omega_{0} \cos \omega t$. We refer to $\Omega_{0}=\mathcal{F}_{z_{0}} / \hbar$ as the Rabi frequency, which quantifies the efficiency of the driving mechanism and determines the Rabi time $\tau=\pi / \Omega_{0}$. This driving force competes with the spin relaxation, characterized by the energy relaxation and quantum phase relaxation times, $T_{1}$ and $T_{2}$, as described by the Bloch equations [26].

Within this approximation, the steady-state solution for the population difference between the ground and excited states is given by the resonance curve $P_{0}-P_{1}=\tanh \left(\frac{\hbar \omega_{0}}{2 k_{B} T}\right)$ $\left(1-\frac{\Omega_{0}^{2} T_{1} T_{2}}{1+\left(\omega-\omega_{0}\right)^{2} T_{2}^{2}+\Omega_{0}^{2} T_{1} T_{2}}\right)$, where $P_{1}$ and $P_{0}$ are the occupations of the ground and excited states, respectively, $k_{B}$ is Boltzmann's constant, $T$ is the temperature, and the first term on the right-hand side is the thermal equilibrium solution. Together with $T_{1}$ and $T_{2}, \Omega_{0}$ is critical to assess by how much $P_{1}-P_{0}$ departs from its equilibrium value. The detection of the previous population imbalance can be accounted for a magnetoresistive mechanism between the surface atom and the magnetic tip, although nonequilibrium effects could be relevant and may deserve future attention [22].

\section{MICROSCOPIC MODELING}

We now elaborate on the microscopic nature of the operator $\mathcal{F}=\frac{\partial \mathcal{H}}{\partial z}$ relevant for the three mechanisms under discussion. For the crystal field mechanism [6], the vertical displacement of the Fe adatom, $z(t)$ modifies the crystal field created by the four closest Mg ions on the surface [see Fig. 1(b)], which in turn modulate the quartic term that allows direct mixing between $L_{z}= \pm 2$ [27]. The interatomic exchange that arises from the overlap of the tails of the atomic orbitals decays exponentially but can be very large at short distances. In the following, we parametrize $J(z)=J_{0} e^{-z / \ell}$, with $\ell=0.06 \mathrm{~nm}$ [20] and $J_{0}=2 \mathrm{meV}[19,20]$, assuming the tip-Fe distance is $d_{\text {Fe-Tip }}=0.6 \mathrm{~nm}$ and $\left\langle\vec{S}_{T}\right\rangle=2 \hbar$.

The rf bias can induce a Rabi oscillation by means of the three different mechanisms, crystal field [Eq. (3)], exchange [Eq. (2)], and dipolar [Eq. (4)]. The Rabi frequency for all mechanisms depends on the amplitude of the oscillation [Eq. (5)], that is modulated by the ac bias. Therefore, the mechanism responsible of the oscillation can be determined by comparing the relative sizes of the Rabi forces $\mathcal{F}_{J}, \mathcal{F}_{\mathrm{CF}}$, and $\mathcal{F}_{\text {dip }}$. For the exchange mechanism, the exponential dependence of the exchange coupling implies that the prefactor in Eq. (2) takes a value $\frac{\partial J(z)}{\partial z}\left\langle\vec{S}_{T}\right\rangle=66.7 \mathrm{meV} / \mathrm{nm}$. The prefactor for the dipolar mechanism as given by Eq. (4) yields $\mu_{B} \frac{\partial B_{x}^{\text {Tip }}}{\partial z}=0.02 \mathrm{meV} / \mathrm{nm}$, much smaller than the exchange mechanism and therefore negligible for typical tip-Fe distances for ESR. Finally, the prefactor for the crystal field mechanism in Eq. (3) requires knowledge of the local crystal field of Fe. For that matter, we need a spin Hamiltonian for $\mathrm{Fe}$ on $\mathrm{MgO}$ and, importantly, we need to know how it depends on $z_{0}$.

\section{A. First principles calculation of the spin Hamiltonian}

We derive it starting from a density functional theory (DFT) calculation [28] for the system, following the same procedure described in previous works [23,24]. We build a few-level model for the electrons in the $d$ orbitals of $\mathrm{Fe}$, including the crystal field, spin-orbit coupling and electron-electron interaction and we solve it by numerical diagonalization. The crystal field part of the Hamiltonian is obtained from the representation of the DFT Hamiltonian in the basis of maximally localized Wannier orbitals [29,30] $\mathcal{H}_{\mathrm{CF}}(z)=$ $D_{W}(z) l_{z}^{2}+F_{W}(z)\left(l_{x}^{4}+l_{y}^{4}\right)$, where $D_{W}(z)$ and $F_{W}(z)$ are crystal field parameters that depend on the vertical coordinate of the surface Fe atom $z$. On top of that, we add the spin-orbit coupling operator $\mathcal{H}_{\mathrm{SOC}}=\lambda_{\mathrm{SOC}} \vec{l} \cdot \vec{s}$ with $\lambda_{\mathrm{SOC}}=35 \mathrm{meV}$, the Zeeman term $\mathcal{H}_{B}=\mu_{B} \vec{B} \cdot(\vec{l}+2 \vec{s})$, and the electron-electron Coulomb interaction in the $d$ shell.

Importantly, the Wannierization procedure permits us to compute both the coefficients $D_{W}$ and $F_{W}$ and how they change with the vertical Fe displacement $z$. For equilibrium $z=$ 0 , we find $F_{W}=-10 \mathrm{meV},{ }^{1} D_{W}=-290 \mathrm{meV},\left.\frac{\partial F_{W}}{\partial z}\right|_{z=0}=$ $280 \mathrm{meV} / \mathrm{nm}$. In particular, we also find that the two lowest energy states $|0\rangle$ and $|1\rangle$ of the Hamiltonian eigenstates of $\mathcal{H}_{0} \equiv \mathcal{H}(z)$ have a strong overlap with the states with quantum numbers $\left|L=2, S=2, L_{z}= \pm 2, S_{z}= \pm 2\right\rangle$. This is why the energy difference $\Delta$ is very sensitive to the application of an off-plane field $B_{z}$ and quite insensitive to in-plane components, $B_{x}, B_{y}$.

The results obtained with our method confirm the phenomenological Hamiltonian describing the low-energy multielectronic states for six electrons in the $d$ levels of $\mathrm{Fe}$, in the crystal field of the $\mathrm{MgO}(100)$ surface, proposed by Baumann et al. [6,27]. In particular, the low-energy sector of the Hamiltonian can be parametrized with $\mathcal{H}(z)=D L_{z}^{2}+$ $F\left(L_{+}^{4}+L_{-}^{4}\right)+\Lambda \vec{L} \cdot \vec{S}+\mu_{B} \vec{B} \cdot(\vec{L}+2 \vec{S})$, where $L_{a}$ are the many-body angular momentum operators in the subspace $L=2$ and $\vec{S}$ are spin operators in the $S=2$ subspace, in both cases complying with atomic Hund's rules. By fitting the energies and orbital expectation values of the lowest five states between the multiplet and spin Hamiltonians, we find the relations $D=-160 \mathrm{meV},{ }^{3} F=-2 \mathrm{meV}$, and $\Lambda=-11 \mathrm{meV}$, that permit us to connect the DFT calculation with the spin model in a simple manner.

\section{B. Calculation of Rabi matrix elements}

The derivation of the atomic spin Hamiltonian from DFT permits us to compute the relevant matrix elements for the three

\footnotetext{
${ }^{1}$ Sign change in $F_{W}$ is equivalent to a $45-$ deg rotation.

${ }^{2}$ Small variations in $D_{W}$ do not influence the low-energy spectra.

${ }^{3}$ This effective value can show variations if charge fluctuation was considered due to coupling with the underneath oxygen $p_{z}$ orbital, giving a more accurate comparison with the XMCD spectra in Ref. [27].
} 


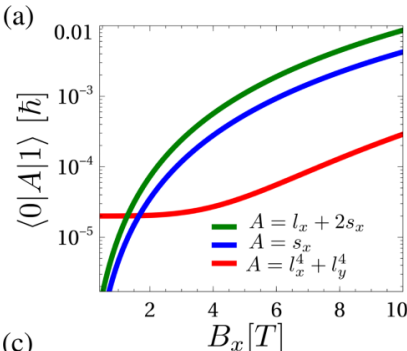

(c)

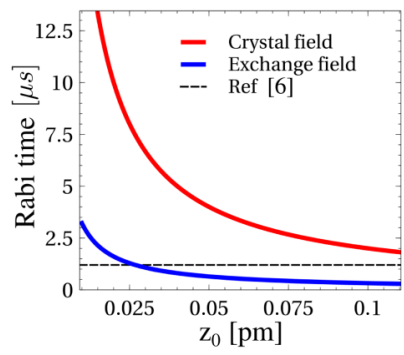

(b)

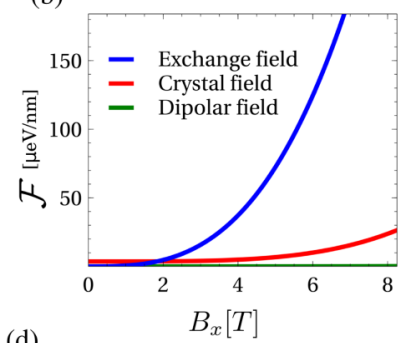

(d)

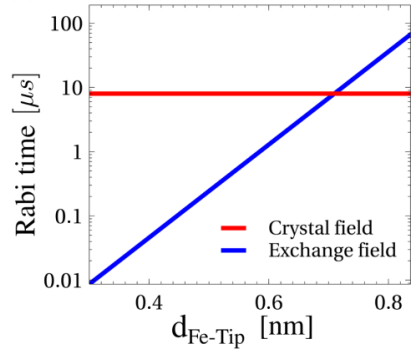

FIG. 2. (a) Matrix elements between the ground and first excited states for the square field perturbation, in-plane Zeeman perturbation, and in-plane exchange perturbation. With the previous matrix elements and the dependence of the Hamiltonian with $z$, the Rabi force can be calculated (b), yielding that the strongest contribution is the exchange mechanism. The Rabi time can be calculated from the Rabi frequency Eq. (5) provided the displacement $z_{0}$ is known, shown in panel (c). The exponential dependence of the exchange field shows that the exchange Rabi time depends on the Fe-tip distance, while the crystal field mechanism is assumed to be independent (d).

mechanisms [Fig. 2(a)], as well as the Rabi forces [Fig. 2(b)]. The matrix elements in Fig. 2(a) show that the biggest offdiagonal terms correspond to the spin operator $s_{x}$ rather than the crystal field operator $l_{x}^{4}+l_{y}^{4}$ at finite in-plane magnetic fields, giving an advantage to the exchange over the crystal field mechanism. When the full Rabi force is calculated, Fig. 2(b), it is obtained that the exchange remains the leading mechanism, followed by the crystal field, whereas the dipolar contribution is nearly negligible. The role of the in-plane magnetic field (applied along the $x$ axis) is to mix the wave functions of $|0\rangle$ and $|1\rangle$ with eigenstates with different $S_{z}$, which finally enables the transitions between them.

The actual value of the Rabi frequency depends on the magnitude of the displacement $z_{0}$. First, we take the value of $z_{0}$ as a free parameter and show how the Rabi time depends on it. In Fig. 2(c), we show the Rabi time as a function of the Fe displacement $z$ for the crystal field and exchange mechanisms, as well as the experimental value as a dashed line. The value of the Fe displacement that would yield a Rabi time comparable to the experimental one of $1.2 \mu s$ [6] would be around $z=0.025 \mathrm{pm}$.

\section{Mechanical modulation of the Fe position}

In the following, we estimate the magnitude of the vertical displacement assuming equilibrium between the electric force $F_{\mathrm{el}}=q_{\mathrm{atom}} E_{\mathrm{RF}}(t)$ and the spring constant $F_{\text {res }}=-k z$, where $k$ is the restoring force. As the driving frequency is in the $\mathrm{GHz}$ range, much smaller than the standard frequency of stretching
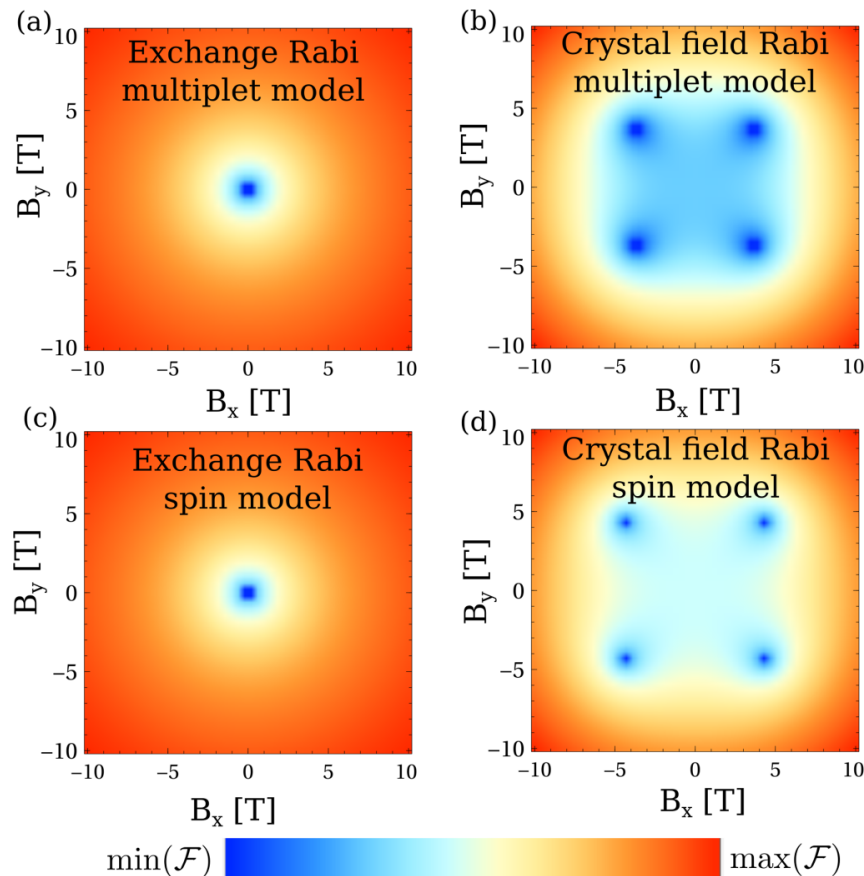

$\min (\mathcal{F})$ $\max (\mathcal{F})$

FIG. 3. Rabi forces for the exchange [panels (a) and (c)] and crystal field [panels (b) and (d)] ESR mechanisms, as a function of the two components of the in-plane field. Panels (a) and (b) correspond to the multiplet model and panels (c) and (d) correspond to the spin model. The crystal field contribution shows nodes when the magnetic field is applied in the (11) directions, whereas the exchange mechanism is isotropic. Such symmetry difference allows us to determine the leading mechanism experimentally.

modes, $\omega_{\mathrm{Fe}}=\sqrt{\frac{k}{M_{\mathrm{Fe}}}}$, in the THz, we can assume that the atom is always at the instantaneous equilibrium position

$$
z(t)=\frac{q_{\mathrm{atom}}}{k} \frac{V_{\mathrm{RF}}(t)}{d}
$$

with $d$ being the decay distance of the electric field, on the order of the Fe-tip distance. DFT calculations yield a value of $k \approx 600 \mathrm{eV} / \mathrm{nm}^{2}$, that for $q=2 e\left(\mathrm{Fe}^{2+}\right), d=d_{\text {Tip-Fe }}=$ $0.6 \mathrm{~nm}$, and the experimental $V_{R F}=8 \mathrm{meV}$ [6] gives a value for the Fe displacement of $z=0.044 \mathrm{pm}$, comparable with the one needed for the Rabi time associated with the exchange field. ${ }^{4}$ For reference, the iron oxide Young modulus [31] would give $z=0.11 \mathrm{pm}$. We note that the previous estimate would show sizable variations if noninteger charging of $\mathrm{Fe}(q \leqslant 2)$ or a larger voltage drop length $\left(d>d_{\text {Tip-Fe }} \mathrm{nm}\right)$ are considered.

\section{Symmetry properties of the Rabi frequency}

We now propose an experimental test to infer which of the proposed mechanisms is actually driving the spins in the case

\footnotetext{
${ }^{4}$ It must be noted that although the zero point spread $\left(\frac{\hbar}{M_{\mathrm{Fe}} \omega}\right)^{1 / 2} \simeq$ $8 \mathrm{pm}$ is larger than the piezoelectric displacement, it does not efficiently couple to the spin because the oscillation frequency of $\langle z(t) z(0)\rangle$, given by $\hbar \omega_{F e}$, is three orders of magnitude larger than the spin resonance frequency.
} 
of Fe on $\mathrm{MgO}$, based on the dependence of the Rabi energy on the orientation of the in-plane magnetic field. For that matter, we plot the Rabi forces, both for the exchange and crystal field mechanisms, as functions of $B_{x}$ and $B_{y}$ (Fig. 3). For reference, the states are computed both with the spin model and with the full multiplet calculation. For the exchange mechanism, we obtain quite isotropic behavior, as expected from the scalar nature of this interaction. In contrast, for the crystal field mechanism, the map reflects the $C_{4}$ local symmetry, developing nodes in the (11) direction [Figs. 3(b) and 3(d)]. This same analysis could be carried out to distinguish the relevant mechanism for other atoms deposited on different surfaces. In case the dominant mechanism is the crystal field, this makes it possible to determine the local distortions by observing the symmetry of the Rabi response with the in-plane magnetic fields.

\section{CONCLUSION}

We have shown that a modulated exchange coupling between a surface atom and a magnetic STM tip is an efficient mechanism to induce electron paramagnetic resonance in the surface spin by inducing a Rabi oscillation between the two lowest states. Based on DFT and multiplet calculations, we show that this mechanism is necessary to account for the Rabi time measured in the $\mathrm{Fe}$ on $\mathrm{MgO}$ experiment [6]. Importantly, the exchange-driven mechanism shows that the ESR technique is way more general and could be realized in systems that do not have a specific crystal field and a sizable spin-orbit coupling.

\section{ACKNOWLEDGMENTS}

J.L.L. and J.F.R. acknowledge financial support by MarieCurie-ITN 607904 SPINOGRAPH and COMPETE 2020FCT Portugal PTDC/FIS-N AN/4662/2014 (016656). J.F.R. acknowledges financial support by MEC-Spain (FIS201347328-C2-2-P and MAT2016-78625-C2). A.F. acknowledges CONICET (PIP11220150100327) and FONCyT (PICT-20122866). We thank F. Delgado for useful discussions. We especially thank A. Heinrich, C. Lutz, and K. Yang for very useful feedback on their experiment.

\section{APPENDIX}

\section{Dependence of the resonance frequency with the STM tip distance}

Our theory can also account for the experimental observation of small variations of the resonance frequency on the tip-Fe $d$ distance for $\mathrm{Fe}$ on $\mathrm{MgO}$ [6] that have nonmonotonic dependences. Whereas the driving forces are dominated by the in-plane component of the tip magnetic moment, the much smaller off-plane component adds to the effective magnetic field $\mathcal{B}_{z}(d)$ that controls the resonant frequency of the surface spin, on account of its large off-plane magnetic anisotropy [6].
This effective field $B_{z}^{\text {eff }}$ is the sum of the actual magnetic field $B_{z}$ plus the exchange and dipolar contributions:

$$
B_{z}^{\text {eff }}=B_{z}-\frac{\chi J\left(d_{\mathrm{Fe}-\mathrm{tip}}\right)}{\mu_{B}}\left\langle S_{z}^{\mathrm{tip}}\right\rangle+\frac{\mu_{0}}{4 \pi} \frac{2\left\langle\bar{S}_{z}^{\mathrm{tip}}\right\rangle}{d_{\mathrm{Fe}-\mathrm{ip}}^{3}},
$$

where $\chi \approx 0.4$ accounts for the fact that exchange coupling couples only to $S_{z}$, whereas an effective magnetic field couples to $2 S_{z}+L_{z}$. It is worth noting that in the case of multiple Fe atoms in the STM tip, the effective expectation values on the dipolar $\left\langle\bar{S}_{z}^{\text {tip }}\right\rangle$ and exchange $\left\langle S_{z}^{\text {tip }}\right\rangle$ contribution may be different, since only the closest atom in the tip would contribute to exchange interaction, but all of them contribute to dipolar interaction.

Whereas tip-surface exchange is antiferromagnetic [19,20], dipolar interaction is ferromagnetic along the off-plane direction. At short tip-surface distance, exchange dominates, whereas at longer distance, dipolar coupling prevails. This competition leads to of $\mathcal{B}_{z}$ as a function of $d_{\mathrm{Fe}-\text { tip }}$, as seen in the experiment.

\section{Role of nuclear spin moment in the ESR peaks}

We comment on the role of nuclear spins. When coupled to a nuclear spin $I$, the otherwise unique electronic spin resonance splits into $2 I+1$ lines, but the total special weight remains the same, so that the visibility of lines is diminished accordingly. In the case of $\mathrm{Fe}$, the most abundant isotope $(91 \%)$ is ${ }^{56} \mathrm{Fe}$, with $I=0$, which accounts for the observation of a single peak ${ }^{5}$. In comparison, the only stable isotope for Co has $I=7 / 2$, which results in eight peaks split by the hyperfine coupling, definitely larger than the reported line width, but each of them diminished by a factor of 8 that hinders their detection.

\section{Other mechanisms}

We briefly discuss other mechanisms that couple the electric field to the surface spins. First, the ac electric field generates an ac magnetic field that couples to the surface spins. We have estimated the magnitude of this field to be in the range of nano-Tesla, so that the resulting Rabi time would be $10^{-6}$ times larger than the one observed experimentally.

In Ref. [22], a mechanism is proposed based on the renormalization of magnetic anisotropy due to exchange interaction with the tunneling electrons. This renormalization is a variant of the one observed experimentally [32] for $\mathrm{Co}$ atoms on a $\mathrm{Cu}_{2} \mathrm{~N}$ surface as well as hydrogenated $\mathrm{Co}$ atoms on boron nitride on $\mathrm{Rh}(111)$ [33], caused by exchange coupling with the surface electrons $[32,34,35]$. The correlation mechanism proposed in Ref. [22] relies on the time modulation of the anisotropy renormalization induced by the exchange with the tunneling electrons.

Finally, in addition to the piezoelectric displacement considered in this work, the electric field also distorts the electronic orbitals of the surface atom and tip atom, that should also result in a modulation of their exchange interaction. Future work should address the magnitude of this effect.

\footnotetext{
${ }^{5}$ We expect that ${ }^{57} \mathrm{Fe}, 2 \%$ abundance and $I=1 / 2$, results in 2 peaks
} 
[1] Y. Manassen, R. J. Hamers, J. E. Demuth, and A. J. Castellano, Jr., Phys. Rev. Lett. 62, 2531 (1989).

[2] C. Durkan and M. Welland, Appl. Phys. Lett. 80, 458 (2002).

[3] T. Komeda and Y. Manassen, Appl. Phys. Lett. 92, 212506 (2008).

[4] A. V. Balatsky, M. Nishijima, and Y. Manassen, Adv. Phys. 61, 117 (2012).

[5] S. Müllegger, S. Tebi, A. K. Das, W. Schöfberger, F. Faschinger, and R. Koch, Phys. Rev. Lett. 113, 133001 (2014).

[6] S. Baumann, W. Paul, T. Choi, C. P. Lutz, A. Ardavan, and A. J. Heinrich, Science 350, 417 (2015).

[7] A. V. Balatsky, Y. Manassen, and R. Salem, Phys. Rev. B 66, 195416 (2002).

[8] L. N. Bulaevskii, M. Hruška, and G. Ortiz, Phys. Rev. B 68, 125415 (2003).

[9] A. Caso, B. Horovitz, and L. Arrachea, Phys. Rev. B 89, 075412 (2014).

[10] D. G. Mitchell, M. Tseitlin, R. W. Quine, V. Meyer, M. E. Newton, A. Schnegg, B. George, S. S. Eaton, and G. R. Eaton, Mol. Phys. 111, 2664 (2013).

[11] A. Blank, C. R. Dunnam, P. P. Borbat, and J. H. Freed, J. Magn. Reson. 165, 116 (2003).

[12] C. Hirjibehedin, C.-Y. Lin, A. Otte, M. Ternes, C. P. Lutz, B. A. Jones, and A. J. Heinrich, Science 317, 1199 (2007).

[13] R. C. Jaklevic and J. Lambe, Phys. Rev. Lett. 17, 1139 (1966).

[14] M. Assig, M. Etzkorn, A. Enders, W. Stiepany, C. R. Ast, and K. Kern, Rev. Sci. Instrum. 84, 033903 (2013).

[15] T. Choi, W. Paul, S. Rolf-Pissarczyk, A. J. Macdonald, F. D. Natterer, K. Yang, P. Willke, C. P. Lutz, and A. J. Heinrich, Nat. Nanotechnology 12, 420 (2017).

[16] F. D. Natterer, K. Yang, W. Paul, P. Willke, T. Choi, T. Greber, A. J. Heinrich, and C. P. Lutz, Nature 543, 226 (2017).

[17] R. Schmidt, C. Lazo, H. H. 1scher, U. H. Pi, V. Caciuc, A. Schwarz, R. Wiesendanger, and S. Heinze, Nano Lett. 9, 200 (2009).

[18] K. Tao, V. S. Stepanyuk, W. Hergert, I. Rungger, S. Sanvito, and P. Bruno, Phys. Rev. Lett. 103, 057202 (2009).

[19] R. Schmidt, C. Lazo, U. Kaiser, A. Schwarz, S. Heinze, and R. Wiesendanger, Phys. Rev. Lett. 106, 257202 (2011).

[20] S. Yan, D.-J. Choi, J. A. Burgess, S. Rolf-Pissarczyk, and S. Loth, Nat. Nanotechnol. 10, 40 (2015).
[21] M. Muenks, P. Jacobson, M. Ternes, and K. Kern, Nat. Commun. 8, 14119 (2017).

[22] P. Berggren and J. Fransson, Sci. Rep. 6, 25584 (2016).

[23] A. Ferrón, F. Delgado, and J. Fernández-Rossier, New J. Phys. 17, 033020 (2015).

[24] A. Ferrón, J. L. Lado, and J. Fernández-Rossier, Phys. Rev. B 92, 174407 (2015).

[25] H. González-Herrero, J. M. Gómez-Rodríguez, P. Mallet, M. Moaied, J. J. Palacios, C. Salgado, M. M. Ugeda, J.-Y. Veuillen, F. Yndurain, and I. Brihuega, Science 352, 437 (2016).

[26] A. Abragam and B. Bleaney, Electron Paramagnetic Resonance of Transition Ions (Oxford University Press, Oxford, UK, 1970).

[27] S. Baumann, F. Donati, S. Stepanow, S. Rusponi, W. Paul, S. Gangopadhyay, I. G. Rau, G. E. Pacchioni, L. Gragnaniello, M. Pivetta, J. Dreiser, C. Piamonteze, C. P. Lutz, R. M. Macfarlane, B. A. Jones, P. Gambardella, A. J. Heinrich, and H. Brune, Phys. Rev. Lett. 115, 237202 (2015).

[28] P. Giannozzi, S. Baroni, N. Bonini, M. Calandra, R. Car, C. Cavazzoni, D. Ceresoli, G. L. Chiarotti, M. Cococcioni, I. Dabo, A. Dal Corso, S. de Gironcoli, S. Fabris, G. Fratesi, R. Gebauer, U. Gerstmann, C. Gougoussis, A. Kokalj, M. Lazzeri, L. Martin-Samos, N. Marzari, F. Mauri, R. Mazzarello, S. Paolini, A. Pasquarello, L. Paulatto, C. Sbraccia, S. Scandolo, G. Sclauzero, A. P. Seitsonen, A. Smogunov, P. Umari, and R. M. Wentzcovitch, J. Phys.: Condens. Matter 21, 395502 (2009).

[29] N. Marzari, A. A. Mostofi, J. R. Yates, I. Souza, and D. Vanderbilt, Rev. Mod. Phys. 84, 1419 (2012).

[30] A. A. Mostofi, J. R. Yates, Y.-S. Lee, I. Souza, D. Vanderbilt, and N. Marzari, Comput. Phys. Commun. 178, 685 (2008).

[31] J. Nicholls, D. Hall, and P. Tortorelli, Mat. High Temp. 12, 141 (1994).

[32] J. C. Oberg, M. R. Calvo, F. Delgado, M. Moro-Lagares, D. Serrate, D. Jacob, J. Fernández-Rossier, and C. F. Hirjibehedin, Nat. Nanotechnol. 9, 64 (2014).

[33] P. Jacobson, T. Herden, M. Muenks, G. Laskin, O. Brovko, V. Stepanyuk, M. Ternes, and K. Kern, Nat. Commun. 6, 8536 (2015).

[34] F. Delgado, C. F. Hirjibehedin, and J. Fernández-Rossier, Surf. Sci. 630, 337 (2014).

[35] D. Jacob and J. Fernández-Rossier, Eur. Phys. J. B 89, 210 (2016). 